TITLE:

\title{
Dynamics of exciton-hole recombination in hole-doped single-walled carbon nanotubes
}

\section{AUTHOR(S):}

Nishihara, Taishi; Yamada, Yasuhiro; Kanemitsu, Yoshihiko

\section{CITATION:}

Nishihara, Taishi ...[et al]. Dynamics of exciton-hole recombination in hole-doped singlewalled carbon nanotubes. Physical Review B 2012, 86(7): 075449.

ISSUE DATE:

2012-08-22

URL:

http://hdl.handle.net/2433/159453

RIGHT:

(C)2012 American Physical Society 
PHYSICAL REVIEW B 86, 075449 (2012)

\title{
Dynamics of exciton-hole recombination in hole-doped single-walled carbon nanotubes
}

\author{
Taishi Nishihara, Yasuhiro Yamada, and Yoshihiko Kanemitsu* \\ Institute for Chemical Research, Kyoto University, Uji, Kyoto 611-0011, Japan \\ (Received 18 April 2012; revised manuscript received 31 July 2012; published 22 August 2012)
}

\begin{abstract}
The exciton decay dynamics in hole-doped single-walled carbon nanotubes (SWCNTs) has been studied by femtosecond pump-probe transient absorption (TA) spectroscopy. By the doping of SWCNTs with holes, a fast decay component with the lifetime of a few picoseconds appears in TA signals, which corresponds to exciton decay through the Auger recombination between an exciton and a hole and the trion formation. We revealed that this exciton decay rate is quantized by the number of holes in a single SWCNT. The number of holes of a hole-doped SWCNT is successfully evaluated on the basis of TA decay dynamics.
\end{abstract}

DOI: 10.1103/PhysRevB.86.075449

PACS number(s): 78.67.Ch, 71.35.Cc, 71.35.Pq, 78.47.jb

\section{INTRODUCTION}

The optical properties of semiconducting single-walled carbon nanotubes (SWCNTs) have been studied extensively from the viewpoints of fundamental physics and potential device applications. ${ }^{1}$ Because electrons and holes are strongly confined in one-dimensional (1D) structures of around $1 \mathrm{~nm}$ in diameter, excitons have extremely large binding energy, enabling them to be stable even at room temperature. ${ }^{2}$ Exciton recombination processes govern the optical properties of SWCNTs, ${ }^{2-5}$ and manipulation of exciton dynamics and the creation of new optical functionalities necessitate the doping of SWCNTs with carriers. ${ }^{6-14}$ Strong quantum confinement of excitons enhances the many-body effects among excitons, electrons, and holes in carrier-doped SWCNTs. Time-resolved transient absorption (TA) and photoluminescence (PL) spectroscopy measurements have revealed that nonradiative Auger recombination of excitons plays a dominant role in the exciton recombination dynamics in SWCNTs. ${ }^{15-20}$ Exciton-exciton and exciton-electron (hole) interactions strongly affect the PL quantum efficiency and device performance. Moreover, a recent study has reported that absorption and PL peaks attributed to 1D excitons are modified by hole doping, and that trions (positively charged excitons, the bound states of the exciton-hole complex) are formed in hole-doped SWCNTs. ${ }^{14}$ It is, however, difficult to estimate the number of holes doped in SWCNTs from optical absorption changes, because sharp absorption peaks are due to stable excitons, but not the van Hove singularities in the joint density of states of the conduction-band electron and the valence-band hole. Given that doping of SWCNTs is essential for device applications, it is necessary to gain a proper understanding of the multipleparticle processes involving excitons and holes to be able to develop SWCNT-based photonics devices.

In this work, we report the exciton recombination dynamics in hole-doped SWCNTs studied by TA spectroscopy using a pump-probe technique. The photon energy of the probe pulse was tuned at the $E_{11}$ transition for excitons of $(7,5)$ SWCNTs. By adding chemical dopant into carbon nanotube solutions, a fast decay component with the lifetime of a few picoseconds appears in TA signals, which corresponds to the exciton decay process determined by the exciton-hole Auger recombination and the trion formation. We revealed that the exciton decay rate is quantized by the hole number in a single SWCNT, and the hole number distribution of SWCNTs in the sample is successfully evaluated on the basis of TA decay curve analyses. We discussed the strong reduction of the exciton absorption peak due to hole doping in conjunction with the exciton-hole interaction in 1D SWCNTs. Our results provide a good opportunity to understand the physics behind the carrier-doping effects on 1D systems.

\section{EXPERIMENT}

Carbon nanotubes synthesized by the CoMoCAT methods, dispersed in toluene solutions with 0.9 wt.\% poly $(9,9$ dioctylfluorenyl-2,7-diyl) (PFO), were used. ${ }^{21}$ The samples were treated as follows: moderate bath sonication for $60 \mathrm{~min}$, vigorous sonication with a tip-type sonicator for $5 \mathrm{~h}$, and ultracentrifugation at $18500 \mathrm{~g}$ for $90 \mathrm{~min}$. Hole doping was achieved using a $p$-type dopant, 2,3,5,6-tetrafluoro-7,7,8,8tetracyanoquinodimethane ( $\left.\mathrm{F}_{4} \mathrm{TCNQ}\right)$, in a $1 \mathrm{mg} / \mathrm{mL}$ solution of toluene. ${ }^{11}$ Transient absorption spectroscopy was performed by a pump-probe technique. A quartz sample cell with a path length of $1 \mathrm{~mm}$ was used for performing TA measurements. The light source was a wavelength-tunable optical parametric amplifier based on a regenerative amplified mode-locked Ti:sapphire laser with a pulse duration of $150 \mathrm{fs}$ and a repetition rate of $1 \mathrm{kHz}$. The pump and probe pulse energies were 1.90 and $1.18 \mathrm{eV}$, tuned to the $E_{22}$ and $E_{11}$ excitons, respectively, for $(7,5)$ nanotubes. The laser spot size on the sample surface was measured carefully by the knife-edge method. All measurements were performed at room temperature.

\section{RESULTS AND DISCUSSION}

Absorption spectra of PFO-dispersed SWCNTs with different $\mathrm{F}_{4}$ TCNQ concentrations are shown in Fig. 1(a). The strong absorption peak at $1.18 \mathrm{eV}$ is attributed to the lowest $E_{11}$ exciton for $(7,5)$ SWCNTs. Strong absorption peaks are not observed for SWCNTs with other chiralities, indicating that our samples are highly enriched in a single chirality. Hole doping causes a reduction in the intensity of the $1.18 \mathrm{eV}$ absorption peak and the formation of a new absorption peak at $1.01 \mathrm{eV}$. This new peak is assigned to the absorption of the trions of $(7,5)$ SWCNTs. ${ }^{14}$ Figure 1 (b) shows the spectrally integrated intensities of the $E_{11}$ excitons and trions as a function of the $\mathrm{F}_{4}$ TCNQ concentration. While the absorption intensity of the trions increases with the $\mathrm{F}_{4} \mathrm{TCNQ}$ concentration, the absorbance of the $E_{11}$ excitons decreases 
(a)

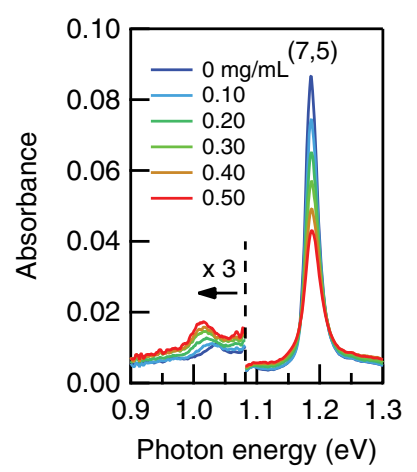

(b)

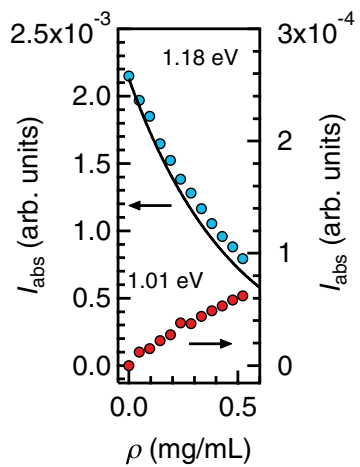

FIG. 1. (Color online) (a) Optical absorption spectra of singlewalled carbon nanotubes with different chemical dopant concentrations. (b) Spectrally integrated absorption intensities at $1.18 \mathrm{eV}\left(E_{11}\right.$ excitons, left axis) and $1.01 \mathrm{eV}$ (trions, right axis) as a function of the dopant concentration $\rho$. The solid curve is the theoretically calculated one.

with the $\mathrm{F}_{4} \mathrm{TCNQ}$ concentration. At $\rho$ of $0.4 \mathrm{mg} / \mathrm{mL}$, the absorption intensity for the $E_{11}$ excitons decreases to a half value compared to the pristine samples.

According to a simple state-filling effect of the valanceband density of state, ${ }^{10}$ this large reduction of absorption intensity suggests the existence of a few tens of holes in a single SWCNT with a length of $100 \mathrm{~nm}$. However, it is accepted that sharp absorption peaks are due to stable excitons, but not the transition between the conduction band and the valence band. ${ }^{4,5}$ Furthermore, in a 1D wire structure, carrier doping causes drastic changes in the absorption spectra near the band edge due to dielectric screening. ${ }^{22}$ These facts indicate that it is difficult to estimate the hole density on the basis of the absorption intensities. Thus, it is necessary to develop a method for estimating the hole density in a single SWCNT.

Figure 2 shows the TA decay dynamics under pump intensities of $4,13,46$, and $160 \mu \mathrm{J} / \mathrm{cm}^{2}$, where the average numbers of photocreated excitons per nanotube, $\langle N\rangle$, are estimated to be $0.1,0.3,1.1$, and 3.7 according to Ref. 23. The photoinduced absorption change $\Delta \alpha$ is negative because of the photobleaching of the $E_{11}$ exciton state. The TA curves show

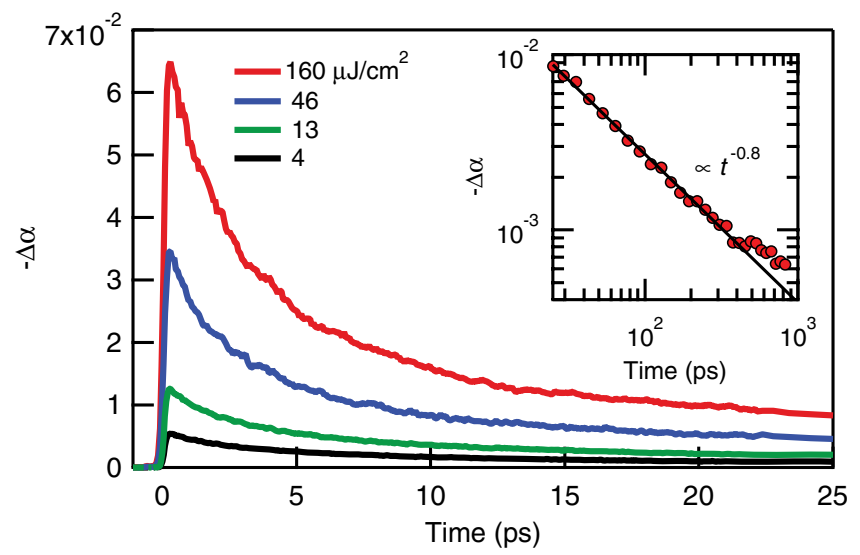

FIG. 2. (Color online) Excitation intensity dependence of TA decay curves in pristine SWCNT samples. The inset shows the TA signals in the long time window.

almost no intensity dependence after $20 \mathrm{ps}$. At long times after photoexcitation, the TA decay is governed by single-exciton dynamics of SWCNTs, which is independent of the initial photogenerated exciton number. The inset of Fig. 2 shows the long-time decay dynamics under an excitation intensity of $160 \mu \mathrm{J} / \mathrm{cm}^{2}$. The relaxation behavior follows the power law $t^{-0.8}$. The power laws of the long-time relaxation dynamics have been reported previously and are ascribed to the 1D exciton diffusion in SWCNTs. ${ }^{24}$

An increase in excitation intensities leads to the appearance of a fast component with a lifetime of $\sim 1 \mathrm{ps}$; this component is attributed to the formation of multiple excitons in a single SWCNT and to the Auger recombination of excitons. ${ }^{15-17}$ It should be emphasized that we observed the ensemble average of TA decay curves, where each nanotube has $N$ excitons $(N=1,2,3, \ldots)$ just after photoexcitation. The fast decay components are ascribed to the multiple exciton recombination $(N \geqslant 2)$, and the exciton lifetimes are quantized by exciton numbers. It is known that such quantized Auger recombination processes are observed in semiconductor nanocrystals. ${ }^{25,26}$

Figure 3 shows the $\mathrm{F}_{4} \mathrm{TCNQ}$-concentration dependence of the TA decay dynamics under an excitation intensity of $4 \mu \mathrm{J} / \mathrm{cm}^{2}$. All the decay curves show nonexponential decay profiles, and the TA signal intensity decreases with an increase of $\mathrm{F}_{4} \mathrm{TCNQ}$ concentration. We found that the TA dynamics shows almost no hole-density dependence after 20 ps. The inset of Fig. 3 shows the TA decay curves normalized at $20 \mathrm{ps.}$ The inset clearly shows no significant difference between two decay curves at long delay times after $20 \mathrm{ps.} \mathrm{An} \mathrm{increase} \mathrm{in}$ the $\mathrm{F}_{4} \mathrm{TCNQ}$ concentration leads to the clear appearance of a fast decay component, but the TA decay profile is independent of the excitation density under our photoexcitation condition. In analogy to the fast decay components appearing under strong photoexcitation in pristine samples (see Fig. 2), this fast component under weak excitations should originate from the many-body effects between excitons and holes such as the exciton-hole Auger recombination and the trion formation, rather than the exciton-exciton Auger recombination. The slow decay component is ascribed to the single exciton recombination, which is almost identical to that of the pristine samples. This result indicates that the sample contains both

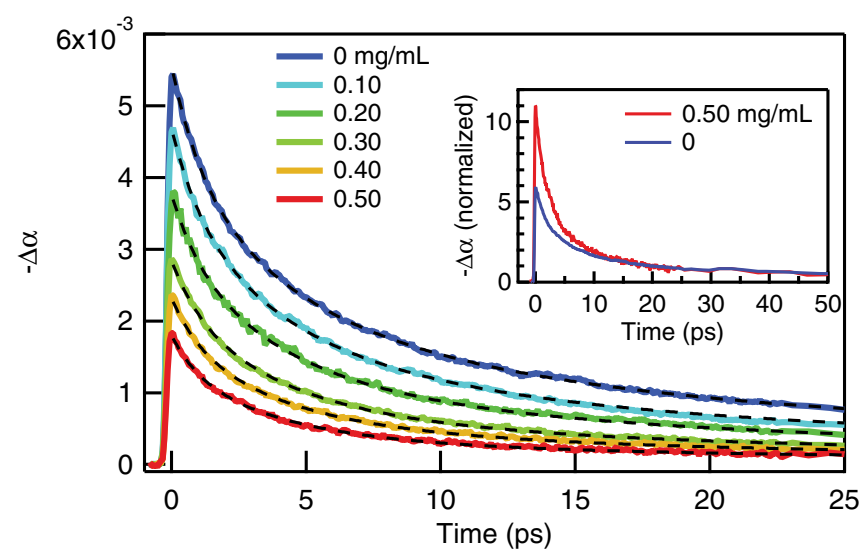

FIG. 3. (Color online) Dopant-concentration dependence of TA decay curves. The inset shows the TA curves normalized at the intensities at 20 ps. The broken curves represent global fits. 
undoped and hole-doped SWCNTs. Note that the observed TA curves are ensemble-averaged over both undoped and holedoped SWCNTs in the sample. Therefore, the experimentally obtained TA signal at the sample with dopant concentration $\rho$ in the picosecond time region, $I_{\mathrm{TA}}^{\mathrm{tot}}(\rho, t)$, can be expressed by the sum of the TA decays of undoped and doped SWCNTs, and is given by

$$
I_{\mathrm{TA}}^{\mathrm{tot}}(\rho, t)=\sum_{n=0}^{\infty} A_{n}(\rho) I_{n}(t),
$$

where $A_{n}(\rho)$ corresponds to the TA signal intensity contributed by SWCNTs doped with $n$ holes in the sample with dopant concentration $\rho$, e.g., $A_{0}$ for undoped SWCNT, $A_{1}$ for SWCNTs doped with one hole, and $A_{2}$ for SWCNTs doped with two holes. $I_{n}(t)$ is the TA decay curve in SWCNTs doped with $n$ holes. Here, $I_{n}(0)$ is assumed to be independent of $n$ and is equal to $1 . A_{n}(\rho)$ is

$$
A_{n}(\rho) \propto \bar{\alpha}_{n} P_{n}(\rho),
$$

where $\bar{\alpha}_{n}$ and $P_{n}(\rho)$ correspond to the absorption coefficient and the concentration fraction of the SWCNTs doped with $n$ holes in the sample with dopant concentration $\rho$. Note that $P_{0}(0)=1$ is satisfied in undoped SWCNTs.

First, we elucidate the TA decay curve of undoped SWCNTs, $I_{0}(t)$, to discuss the hole doping effects on exciton decay dynamics in hole-doped SWCNTs. The TA decay curves of undoped SWCNTs are nonexponential and approximately reproduced by triple exponential functions in the time range between 0 and $100 \mathrm{ps},{ }^{27,28}$ although the decay curves at long delay times are described by the power law. We obtained the three lifetimes of 1.2, 6.1, and 38.2 ps. Using these values and Eq. (1), we performed the global fitting of the TA decay curves in hole-doped samples. For hole-doped samples, the TA decay curves are well reproduced by the sum of the TA decay curve of undoped SWCNTs and two single-exponential functions: $A_{0} I_{0}(t)+A_{1} \exp \left(-t / \tau_{1}\right)+A_{2} \exp \left(-t / \tau_{2}\right)$. The fitting results are shown as broken curves in Fig. 3. This finding shows that new exponential-decay channels appear in hole-doped SWCNTs, and two lifetimes $\tau_{1}$ and $\tau_{2}$ correspond to two-particle interactions between an exciton and a hole in one-hole-doped (ex-h) and two-hole-doped (ex-2h) SWCNTs, respectively. Because of the 1D motion of excitons, the exciton-hole scattering occurs between an exciton and the nearest holes: one or two holes dominate the exciton decay dynamics. Here, we obtained that $\tau_{1}$ and $\tau_{2}$ are 3.8 and $1.0 \mathrm{ps}$, respectively. At long delay times, the TA decay is independent of the doped hole number in SWCNTs.

It should be noted that the hole-number-dependent TA dynamics in doped SWCNTs is quite similar to the quantized Auger recombination in semiconductor nanocrystals, where the exciton population dynamics is sensitive to the number of excitons in a single nanocrystal. ${ }^{25,26}$ Thus, we can extract single-exponential exciton-hole dynamics using the simple subtraction procedure. According to the simple subtraction procedures proposed in Ref. 25 , we subtract the low-concentration TA curve from the high-concentration TA curve, and we obtain the exciton lifetimes in hole-doped SWCNTs. Figure 4 summarizes the decay curves derived from a simple subtraction procedure. The subtracted decay curves

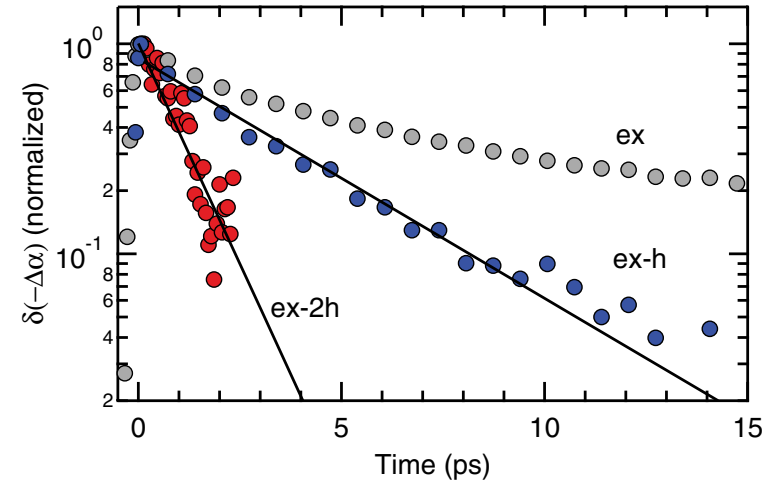

FIG. 4. (Color online) TA curves obtained by the simple subtraction procedure: Decay curves due to single exciton recombination (ex), and to exciton-hole interactions in one-hole-doped (ex-h) and two-hole-doped (ex-2h) SWCNTs.

follow single exponential functions, corresponding to the ex-h and ex-2h lifetimes. These lifetimes are also obtained from the global fitting of the decay curves in Fig. 3. We successfully determined the exciton lifetimes in the hole-doped SWCNTs by using the simple model.

The fitting parameters, $A_{0}, A_{1}$, and $A_{2}$, for the TA decay curves are summarized in Fig. 5(a). The parameter $A_{0}$ for undoped SWCNTs decreases with an increase of $\mathrm{F}_{4} \mathrm{TCNQ}$ concentration, indicating the reduction of undoped SWCNT number in the samples. From the $\rho$ dependence of $A_{0}$, we can calculate the average number of holes per a SWCNT, $\langle n\rangle$, in the case that the probability distribution of the number of doped holes follows the Poisson distribution $P_{n}(\rho)=\langle n\rangle^{n} e^{-\langle n\rangle} / n$ !. Figure 5(b) shows $\langle n\rangle$ as a function of $\mathrm{F}_{4} \mathrm{TCNQ}$ concentration, $\rho$. It can be seen that $\langle n\rangle$ scales linearly with the $\mathrm{F}_{4} \mathrm{TCNQ}$ (a)

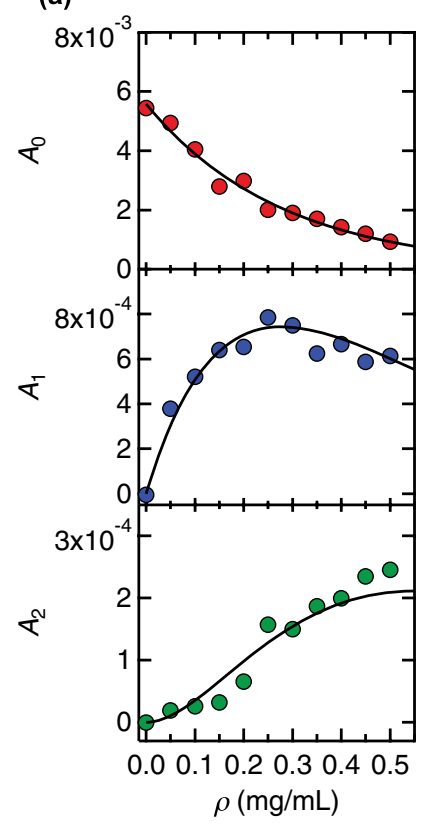

(b)

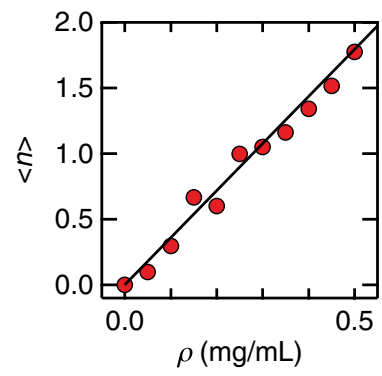

(c)

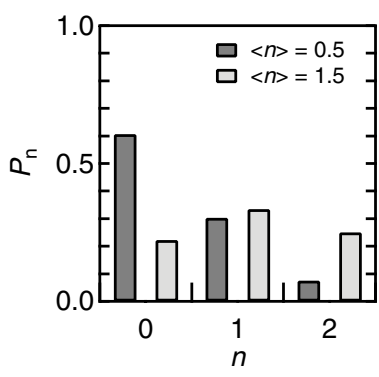

FIG. 5. (Color online) (a) Dopant-concentration dependence of the fitting parameters $A_{0}, A_{1}$, and $A_{2}$. (b) The average number of doped holes, $\langle n\rangle$, evaluated using the Poisson distribution. (c) The hole-number distribution in the SWCNT samples $P_{n}(\rho)$ with the average hole numbers $\langle n\rangle$ of 0.5 and 1.5. 
concentration. This result supports that our model described in Eqs. (1) and (2) is appropriate for the evaluation of hole number in a single SWCNT. It is noteworthy that $\langle n\rangle$ is below 2.0 at the maximum $\mathrm{F}_{4}$ TCNQ concentration in our experiments, indicating that most of the SWCNTs are completely devoid of holes or have just a few of them [see Fig. 5(c)]. We found a new method for evaluating the number of holes in SWCNTs based on the simple model.

The parameter $A_{1}$ for SWCNTs doped with one hole increases with an increase of $\mathrm{F}_{4}$ TCNQ concentration at low $\mathrm{F}_{4}$ TCNQ concentrations, and it shows the maximum value at around $0.3 \mathrm{mg} / \mathrm{mL}$, which corresponds to $\langle n\rangle=1$ estimated by the linear relation of $\langle n\rangle$ and $\rho$ [see Fig. 5(b)]. This is consistent with the fact that the fraction of SWCNTs doped with one hole, $P_{1}(\rho)$, becomes the maximum at $\langle n\rangle=1$. On the other hand, the parameter $A_{2}$ for SWCNTs doped with two holes increases more gradually with $\mathrm{F}_{4} \mathrm{TCNQ}$ concentration, compared to the case of $A_{1}$. This is because the fraction of SWCNTs containing two holes is small at low $\mathrm{F}_{4}$ TCNQ concentrations below $\langle n\rangle=2$. We calculated the concentration dependence of the $A_{0}, A_{1}$, and $A_{2}$ using Eq. (2) and the Poisson distribution of the hole numbers, where the fitting parameters are $\bar{\alpha}_{1}$ and $\bar{\alpha}_{2}$. In Fig. 5, fitting results are shown as solid curves, reproducing the experimental results well. The best-fitting parameters are $\bar{\alpha}_{1} / \bar{\alpha}_{0}=0.36$ and $\bar{\alpha}_{2} / \bar{\alpha}_{0}=0.14$. These results clearly show that our simple model can explain the global features of the exciton dynamics in the hole-doped SWCNTs.

Finally, we discuss the reduction of the lowest exciton absorption peak due to hole doping, as shown in Fig. 1. Using the values of $\bar{\alpha}_{1} / \bar{\alpha}_{0}=0.36$ and $\bar{\alpha}_{2} / \bar{\alpha}_{0}=0.14$, we calculated the absorption intensities of the $E_{11}$ excitons in the hole-doped samples, $\alpha(\rho)=\sum_{n=0}^{2} \bar{\alpha}_{n} P_{n}(\rho)$. The calculated result is shown by the solid curve in Fig. 1(b). We found that the calculated result is in good agreement with the experimental observation. This result clearly shows that the exciton absorption is strongly reduced even in very low hole densities of $\sim 10^{5} \mathrm{~cm}^{-1}$, which is estimated from the average SWCNTs length of a few hundred nanometers. ${ }^{29}$ Such a large reduction of the exciton absorption peak cannot be explained by a simple state-filling effect of the valance-band density of state: ${ }^{10}$ the change in optical absorption is considered to be less than $1 \%$ in the sample doped with $10^{5} \mathrm{~cm}^{-1}$ holes. The exciton-hole interaction modifies the exciton absorption of the hole-doped SWCNTs: a single hole reduces the exciton absorption peak intensity due to the redistribution of the oscillator strength of excitons. ${ }^{14,30}$ Similar results are also observed in GaAs 1D quantum wires: At low carrier densities $\left(\sim 10^{5} \mathrm{~cm}^{-1}\right)$, the formation of negative trions (exciton-electron bound state) strongly reduces the optical absorption of excitons. ${ }^{22}$ In hole-doped SWCNTs, the absorption peak for trions appears at $1.01 \mathrm{eV}$, and the anticorrelation in optical absorption between excitons and trions is observed as a function of dopant concentration, as shown in Fig. 1. These observations indicate that the exciton absorption and dynamics in hole-doped 1D structures are dominated by exciton-hole interactions such as Auger recombination and the trion formation.

\section{CONCLUSION}

In conclusion, we studied the exciton recombination dynamics in hole-doped SWCNTs by TA spectroscopy using a pump-probe technique. By the doping of SWCNTs with holes, fast decay components with the lifetimes of a few picoseconds appear in TA signals. We evaluated the quantized recombination process between an exciton and holes. We successfully estimated the number of holes in hole-doped SWCNTs on the basis of the exciton decay dynamics. Optical spectra and dynamics are influenced by exciton-hole interactions enhanced by the strong confinement of an exciton and holes in 1D structures.

\section{ACKNOWLEDGMENTS}

Part of this work was supported by KAKENHI (20104006), MEXT Project of Integrated Research on Chemical Synthesis, The Sumitomo Electric Industries Group CSR Foundation, and JST-CREST.

\footnotetext{
*Author to whom all correspondence should be addressed: kanemitu@scl.kyoto-u.ac.jp

${ }^{1}$ See, for example, A. Jorio, G. Dresselhaus, and M. S. Dresselhaus, Carbon Nanotubes: Advanced Topics in the Synthesis, Structure, Properties and Applications (Springer, Berlin, 2008).

${ }^{2}$ As a review, see Y. Kanemitsu, Phys. Chem. Chem. Phys. 13, 14879 (2011), and references therein.

${ }^{3}$ T. Ando, J. Phys. Soc. Jpn. 66, 1066 (1997).

${ }^{4}$ F. Wang, G. Dukovic, L. E. Brus, and T. F. Heinz, Science 308, 838 (2005).

${ }^{5}$ J. Maultzsch, R. Pomraenke, S. Reich, E. Chang, D. Prezzi, A. Ruini, E. Molinari, M. S. Strano, C. Thomsen, and C. Lienau, Phys. Rev. B 72, 241402 (2005).

${ }^{6}$ M. S. Strano, C. B. Huffman, V. C. Moore, M. J. O'Connell, E. H. Haroz, J. Hubbard, M. Miller, K. Rialon, C. Kittrell, S. Ramesh, R. H. Hauge, and R. E. Smalley, J. Phys. Chem. B 107, 6979 (2003).
}

${ }^{7}$ J. A. Misewich, R. Martel, Ph. Avouris, J. C. Tsang, S. Heinze, and J. Tersoff, Science 300, 783 (2003).

${ }^{8}$ T. Takenobu, T. Takeno, M. Shiraishi, Y. Murakami, M. Ata, H. Kataura, Y. Achiba, and Y. Iwasa, Nat. Mater. 2, 683 (2003).

${ }^{9}$ G. N. Ostojic, S. Zaric, J. Kono, M. S. Strano, V. C. Moore, R. H. Hauge, and R. E. Smalley, Phys. Rev. Lett. 92, 117402 (2004).

${ }^{10}$ G. Dukovic, B. E. White, Z. Zhou, F. Wang, S. Jockusch, M. L. Steigerwald, T. F. Heinz, R. A. Friesner, N. J. Turro, and L. E. Brus, J. Am. Chem. Soc. 126, 15269 (2004).

${ }^{11}$ M. J. O’Connell, E. E. Eibergen, and S. K. Doorn, Nat. Mater. 4, 412 (2005).

${ }^{12}$ L. Cognet, D. A. Tsyboulski, J.-D. R. Rocha, C. D. Doyle, J. M. Tour, and R. B. Weisman, Science 316, 1465 (2007).

${ }^{13}$ M. Steiner, M. Freitag, V. Perebeinos, A. Naumov, J. P. Small, A. A. Bol, and P. Avouris, Nano Lett. 9, 3477 (2009). 
${ }^{14}$ R. Matsunaga, K. Matsuda, and Y. Kanemitsu, Phys. Rev. Lett. 106, 037404 (2011).

${ }^{15}$ F. Wang, G. Dukovic, E. Knoesel, L. E. Brus, and T. F. Heinz, Phys. Rev. B 70, 241403 (2004).

${ }^{16}$ L. Valkunas, Y.-Z. Ma, and G. R. Fleming, Phys. Rev. B 73, 115432 (2006).

${ }^{17}$ L. Huang and T. D. Krauss, Phys. Rev. Lett. 96, 057407 (2006).

${ }^{18}$ R. M. Russo, E. J. Mele, C. L. Kane, I. V. Rubtsov, M. J. Therien, and D. E. Luzzi, Phys. Rev. B 74, 041405 (2006).

${ }^{19}$ L. Lüer, S. Hoseinkhani, D. Polli, J. Crochet, T. Hertel, and G. Lanzani, Nat. Phys. 5, 54 (2009).

${ }^{20}$ Y. Murakami and J. Kono, Phys. Rev. Lett. 102, 037401 (2009).

${ }^{21}$ A. Nish, J. Y. Hwang, J. Doig, and R. J. Nicholas, Nat. Nanotech. 2, 640 (2007).

${ }^{22}$ T. Ihara, Y. Hayamizu, M. Yoshita, H. Akiyama, L. N. Pfeiffer, and K. W. West, Phys. Rev. Lett. 99, 126803 (2007).
${ }^{23}$ L. J. Carlson, S. E. Maccagnano, M. Zheng, J. Silcox, and T. D. Krauss, Nano Lett. 7, 3698 (2007).

${ }^{24}$ Z. Zhu, J. Crochet, M. S. Arnold, M. C. Hersam, H. Ulbricht, D. Resasco, and T. Hertel, J. Phys. Chem. C 111, 3831 (2007).

${ }^{25}$ V. I. Klimov, A. A. Mikhailovsky, D. W. McBranch, C. A. Leatherdale, and M. G. Bawendi, Science 287, 1011 (2000).

${ }^{26}$ A. Ueda, T. Tayagaki, and Y. Kanemitsu, J. Phys. Soc. Jpn. 78, 083706 (2009).

${ }^{27}$ A. Ueda, K. Matsuda, T. Tayagaki, and Y. Kanemitsu, Appl. Phys. Lett. 92, 233105 (2008).

${ }^{28}$ S. Wang, M. Khafizov, X. Tu, M. Zheng, and T. D. Krauss, Nano Lett. 10, 2381 (2010).

${ }^{29}$ F. Hennrich, R. Krupke, K. Arnold, J. A. Rojas Stu1tz, S. Lebedkin, T. Koch, T. Schimmel, and M. M. Kappes, J. Phys. Chem. B 111, 1932 (2007).

${ }^{30}$ S. M. Santos, B. Yuma, S. Berciaud, J. Shaver, M. Gallart, P. Gilliot, L. Cognet, and B. Lounis, Phys. Rev. Lett. 107, 187401 (2011). 